xiv

\title{
Note on a determinant derived from a triangular array.
}

The array of binomial coefficients written in triangular form has many curious properties, particularly so if alternate rows or alternate columns receive a negative sign. We may consider such a scheme, developed from its top right hand corner, containing $n$ rows and columns.

$$
\begin{array}{llllll}
\ldots & 1 & -1 & 1 & -1 & 1 \\
\ldots & 4 & -3 & 2 & -1 & \\
\ldots & 6 & -3 & 1 & & \\
\ldots & 4 & -1 & & & \\
\ldots & 1 & & & &
\end{array}
$$

By filling the lower right hand triangle with zeros, we obtain a square array which we may call $X$. After the first row any entry here is numerically equal to the sum of the two adjacent entries immediately to the right, level or above it, for this is a fundamental property of such binomial coefficients. If we apply this property to each element of any diagonal running downwards to the right and starting at any position on the top row, we infer that the sum of the elements of three such adjacent diagonals is zero.

Thus if $s_{r}$ is the sum of elements in the $r^{\text {th }}$ diagonal counted from the right we have

$$
\begin{array}{lr}
s_{1}=1, s_{2}=-1, s_{3}=1-1=0, \\
s_{r}+s_{r+1}+s_{r+2}=0 & r>2 .
\end{array}
$$

These sums are terms of a recurring series, and in general

$$
s_{3 k-1}=-1, s_{3 k}=0, s_{3 k+1}=1 .
$$

From this array $X$ we can form its determinant $|X|$ which is always equal to unity, since on expansion only that term corresponding to the hypotenuse of the triangle in the above array is non-zero: also the sign automatically is positive for each value of $n$.

Closely allied with this relatively uninteresting determinant is that with $\lambda$ substracted from each element in the leading diagonal. 
Let us call it $\Delta_{\mu} . \quad$ It is the object of this note to exhibit the periodic behaviour of this $\Delta_{n}$ as $n$ increases. We have

$$
\begin{aligned}
& \Delta_{1}=1-\lambda, \\
& \Delta_{2}=\left|\begin{array}{lr}
-1-\lambda & 1 \\
-1 & -\lambda
\end{array}\right|=1+\lambda+\lambda^{2}=\frac{1-\lambda^{3}}{1-\lambda}, \\
& \Delta_{3}=\left|\begin{array}{lrr}
1-\lambda & -1 & 1 \\
2 & -1-\lambda & . \\
1 & . & -\lambda
\end{array}\right|=1-\lambda^{3}, \\
& \Delta_{4}=\left|\begin{array}{cccc}
-1-\lambda & 1 & -1 & 1 \\
-3 & 2-\lambda & -1 & \cdot \\
-3 & 1 & -\lambda & \cdot \\
-1 & . & . & -\lambda
\end{array}\right|=(1-\lambda)\left(1-\lambda^{3}\right)
\end{aligned}
$$

and in general

$$
\begin{aligned}
& \Delta_{3 k-1}=\frac{\left(1-\lambda^{3}\right)^{k}}{1-\lambda}, \\
& \Delta_{3 k}=\left(1-\lambda^{3}\right)^{k}, \\
& \Delta_{3 k+1}=\left(1-\lambda^{3}\right)^{k}(1-\lambda) .
\end{aligned}
$$

It would be interesting to have a direct proof of this curious result. I have only succeeded in proving it by use of a comparatively advanced theorem in the theory of matrices. Thus if $\lambda$ is once more deleted and the array $X$ be treated as a matrix, then on multiplying row by column the matrix $X^{2}$ is found to be the array $X$ turned upside down

$$
\begin{aligned}
& \begin{array}{rrr} 
& 1 & \ldots \\
-1 & 4 & \ldots
\end{array} \\
& \text {. } 1-36 \quad \ldots \text {. } \\
& \begin{array}{rrrrrr} 
& -1 & 2 & -3 & 4 & \ldots \\
1 & -1 & 1 & -1 & 1 & \ldots
\end{array}
\end{aligned}
$$

while $X^{3}$ formed by multiplying $X$ by $X^{2}$ is the unit matrix with ones in the leading diagonal and zeros elsewhere,

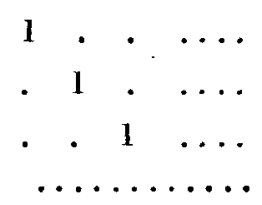


xvi

We can prove these results ${ }^{1}$ by verifying the several elements first of $X^{2}$ and then of $X X^{2}$.

Now the roots $\Delta \mu=0$ are the latent roots of $X$, which are known to be cube roots of those of $X^{3}$, by a theorem due to Sylvester. And since $X^{3}=1$ its latent roots are all equal to unity. Hence the only factors of $\Delta_{n}$ are

$$
(1-\lambda),(\omega-\lambda),\left(\omega^{2}-\lambda\right)
$$

where $1+\omega+\omega^{2}=0$. Thus for certain integral values $p, q$, we must have

$$
\Delta_{n}=c(1-\lambda)^{w}(\omega-\lambda)^{q}\left(\omega^{2}-\lambda\right)^{r} .
$$

Since $\Delta_{n}$ is real, $q=r$, and since $\Delta_{n}=1$ if $\lambda=1$, then $c=1$.

Finally equating the coefficient of $\lambda^{n-1}$ on both sides we obtain

$$
s_{n}=p-q,
$$

where $s_{n}$ is the sum of the leading diagonal terms of the determinant with $\lambda$ suppressed throughout. But it has been shewn that

$$
s_{3 k-1}=-1, s_{3 k}=0, s_{3 k+1}=1
$$

from which we deduce the actual values of $p$ and $q$ and thereby establish results.

Similar results may be deduced by starting with an array where alternate rows have negative signs.

$$
\text { H. W. TuRn BCLL. }
$$

\section{Principal radii of curvature at a point on an ellipsoid.}

The values of the principal radii of curvature $\rho_{1}, \rho_{2}$ at a point on an ellipsoid are $\alpha^{2} / p, \beta^{2} / p$ where $\alpha^{2}, \beta^{2}$ are the squares of the semi-axes of the central section parallel to the tangent plane at the point, and $p$ is the distance from the centre to the tangent plane.

Probably the neatest proof of this theorem is that given in C. Smith's textbook (7th ed. p. 228). The following proof has a certain interest, as it assumes less than the other.

Choose the origin $O$, at the point, take $O Z$ along the inward drawn normal, and $O X, O Y$ parallel to the axes of any section by a

1 "On the matrix square and cube roots of unity." Juurnal of the London Math. Soc., 2 (1927) November. 